\title{
Las torres de las murallas de Segovia
}

\author{
Miguel ÁNGEL MARTín BLANCO \\ Arquitecto \\ martinblanco.miguel@gmail.com
}

\begin{abstract}
Resumen
Las murallas han sido a lo largo de la Historia uno de los elementos fundamentales de los asentamientos humanos, definitorios de la configuración urbana e incluso de la condición de ciudad. Ciudad y muralla, no se entienden el uno sin el otro. En el caso de Segovia, distribuidas por el trazado de la muralla, atendiendo a su morfología y diseño en planta, existen tres tipos de torres, poligonales, rectangulares y circulares. Esta diferenciación no parece tener sentido tratándose de un elemento de carácter militar y sin ninguna finalidad estética, por lo que no encontramos ninguna lógica aparente que lo justifique. En la documentación histórica parece insinuarse una unidad y contemporaneidad en la construcción de todo su trazado, lo cual contraviene esta diferenciación estilística que se observa en el documento construido.

En construcción todo está modulado en base a una unidad de medida, la cual históricamente estaba generalmente relacionada con el cuerpo humano. Estas unidades de medida, estos módulos constructivos históricos, varían de una civilización a otra y de una época a otra. La existencia de distintas tipologías de torres, de diferentes tipos de fabricas, aparejos y de métricas constructivas nos inducen a pensar en la construcción de una muralla efectuada en distintas fases constructivas y no en una obra tan unitaria como parece respaldar la historiografía tradicional.
\end{abstract}

Palabras clave: Segovia; murallas; torres; ciudades amuralladas. 


\title{
Towers of the walledtown of Segovia
}

\begin{abstract}
Defensive walls have been a history along one of the key elements of human settlements, defining urban settings and even city status. Defensive walls and the city did not understand one without the other. In the case of Segovia distributed over the defensive wall, according to their morphology and plant design, there are three types of towers: polygonal, rectangular and circular. This difference does not seem to make sense in the case of a military element and without any aesthetic purpose, so we find no apparent logic to justify it. In historical documentation and apparently there is a contemporary unit in building all their drawing, which is not consistent with this stylistic difference observed in the construction.

The construction is based on a measurement unit, which historically was usually related to the human body. These units of measurement, these historical construction modules, change from one civilization to another and from one era to another. The existence of different types of towers, building materials, canvases and constructive metric, lead us to think about the construction of a defensive wall made in different phases of construction, not as unified as a work seems to support the traditional historiography.
\end{abstract}

Key words: Segovia; citywalls; towers; walledtowns.

Sumario: 1.- Introducción. 2.- Tipología de las torres de las murallas de Segovia 2.1.Sector 1. 2.2.- Sector 2. 2.3.- Sector 3. 3.- Conclusiones. 4.- Referencias bibliográficas.

\section{Introducción.}

La ciudad de Segovia se encuentra situada sobre una roca de forma alargada de Este a Oeste, entre los ríos Eresma al Norte y Clamores al Sur. Su insuperable posición le aseguraba una defensa natural, no obstante, se reforzó con una muralla. Ésta nace y muere en el Alcázar y cuenta con un perímetro superior a los 3.000 metros, que se va adaptando al límite de la peña aprovechando los escarpes naturales del terreno, En su recorrido se dispusieron torres, cubos, edificios y también se abrieron puertas y postigos, aunque en la actualidad no permanecen todos los que fueron, la muralla se conserva bastante completa. 
La muralla es uno de los monumentos mas desconocidos de Segovia. Tradicionalmente se asocia su construcción a la Repoblación ordenada por Alfonso VI, pero es asombrosa la escasez de datos históricos existentes sobre Segovia en estos cruciales años en que se crea el germen de la ciudad actual.

No existe ninguna monografía sobre las murallas de Segovia, ni ninguna publicación que nos hable de su cronología, sus sistemas constructivos, de sus materiales, los procesos constructivos, de su métrica, de su configuración urbana, de los elementos de carácter militar que presenta, etc. Quizá los únicos documentos técnicos de carácter global a este respecto sean el Estudio Geotécnico (Rodríguez Ortiz, 1998) y el Plan Director (Fernández-Longoria Alcántara, 2006) ${ }^{1}$.

Partiendo de la planimetría efectuada en este último documento (ver esquema 1) ya que está más actualizada y se basa en el Estudio Geotécnico, se ha efectuado un intenso trabajo de campo, para la toma de datos sobre el elemento construido, fotos, planos, medición ${ }^{2}$, dibujo, etc ${ }^{3}$. Esto ha permitido seleccionar tres sectores de muralla homogéneos, en los que la métrica es la misma, esto es, las dimensiones de las torres y la modulación de los lienzos, situados todos ellos en la cara sur de la ciudad 4 .

\footnotetext{
${ }^{1}$ Todas las referencias a las torres y lienzos de la muralla se hacen en base a la numeración establecida en este documento. Se ha excluido del estudio el tramo correspondiente al Alcázar, desde la torre 62 hasta la 71 ya que su modulación atiende mas a la presencia del propio edificio y a la singular topografía en que se encuentran.

${ }^{2}$ La mayor parte de la medición de la cara sur, mucho más accesible, se ha realizado por medios propios, con cinta métrica y distanciómetro láser. Para la parte norte, se han empleado, las planimetrías de diferentes proyectos de intervención, los informes arqueológicos y la documentación de final de las obras realizadas. En el resto de los casos, donde no se han realzado obras y de difícil acceso se ha recurrido al plano catastral.

${ }^{3}$ Numerosos recorridos a lo largo de la muralla, para realizar levantamientos fotográficos y lecturas constructivas buscando elementos significativos, reinterpretar fabricas, localizar distintas fases e intervenciones, diferentes aparejos, lapidas y otras piezas reutilizadas y otros elementos singulares, elementos gliptográficos, elementos de carácter militar, localizar vestigios arqueológicos, similitudes y diferencias entre elementos, contrastar noticias previas, sospechas o hipótesis, etc.

${ }^{4}$ En la cara norte, de mucho más difícil acceso, las mediciones no es una herramienta tan útil, debido a la orografía. Durante la construcción, posiblemente, la regularidad en la modulación de trazado se sacrifica en favor de una mejor adaptación a lo abrupto del terreno.
} 
En el Plan Director, se identifican tres tipos de torres, atendiendo a su forma en planta: rectangulares, circulares y poligonales. Tras una profunda revisión se han detectado algunas incorrecciones, de las que enumeramos solo las tres que nos interesan a los efectos de esta comunicación.

- Las torres 49, 59, 60, 61 y $61^{\prime}$ se representan como de planta circular. La torre 49 , es una torre de base rectangular, en la que se han expoliado los sillares esquineros y una reparación posterior ha solventado con mampostería redondeando las esquinas en lugar de restituir su forma original. En las obras realizadas recientemente se ha descubierto el arranque de la número $61^{\prime}$ evidenciándose que se trata de una torre de planta cuadrada que ha sufrido un derrumbe (y posiblemente se expoliaron sus sillares esquineros). Respecto a la torre 61, no hay ningún resto material que indique su presencia en la posición en que se representa en los planos del Plan Director, salvo por modulación, dado que el paño que resultaría entre la 60 y la 61' sería excesivamente largo. En cualquier caso para confirmar su ubicación deberían realizarse excavaciones arqueológicas que permitiesen ubicarla certeramente y conocer su forma real. Por último las torres 59 y 60, arrancan ambas sobre una base rectangular, transformándose en planta circular, aparentemente podría el mismo caso comentado que el de la torre $61^{\prime}$. Solo la torre 59 nos plantea dudas, reconstruida por completo en el siglo XX, y que en un grabado de mediados del XIX, se la aprecia circular, al igual que la 60 (Avrial y Flores 1953: lámina 51).

- Solo se consideran de planta poligonal las torres 82 y 83. Las torres 81,85 , 4 y 6 , se consideran de planta circular. Si bien es cierto que en algunos casos se rematan en su parte superior en forma circular, un examen de sus paramentos nos permite afirmar que en origen eran de traza poligonal, ya que aun se evidencian algunas aristas en los restos de su deteriorada sillería en la parte inferior y que se ha perdido en las partes altas en intervenciones posteriores.

- El quiebro de la muralla donde se sitúa la torre 47, está mal ubicado. Su posición real esta mas hacia el oeste, existiendo una torre, no detectada en el Plan Director, entre la 46 y dicho quiebro que hemos bautizado como $46^{\prime}$. Esta diferencia motiva un importante cambio en la modulación de estos lienzos. 


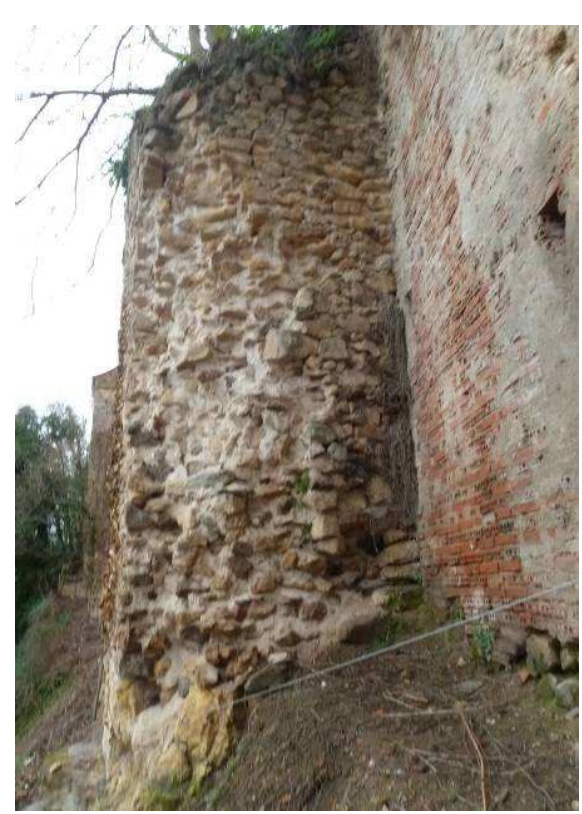

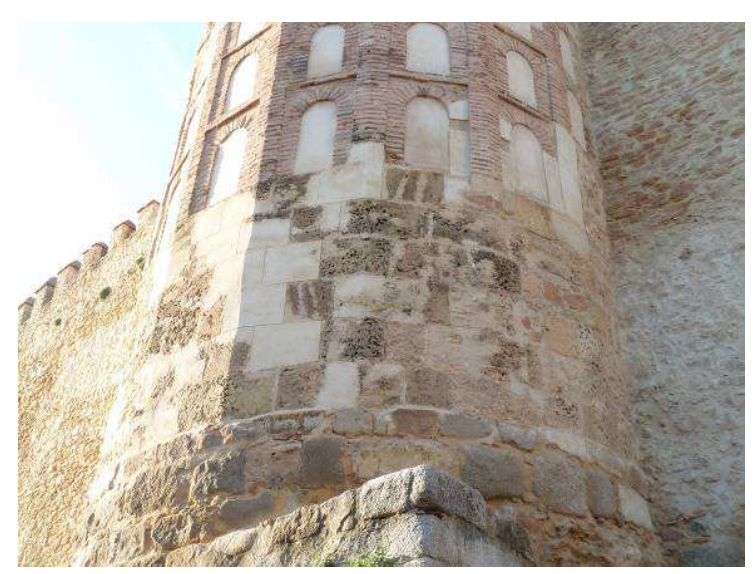

Figs. 1-2. (izq.) Torre 49 de planta rectangular en la que se han expoliado los sillares esquineros, lo que hace aparentar una planta circular. (drch) Torre 4. Se observa la forma poligonal de la torre por la presencia de las aristas de la sillería.

Con estas nuevas consideraciones, se han organizado los lienzos de la muralla en función de los distintos tipos de torres que presenten atendiendo a su forma en planta y a su métrica, centrándose principalmente en las torres, dado que es más fácil encontrar proporcionalidad en su modulación que en los lienzos cuyo trazado y replanteo puede estar condicionado por la orografía. Dado que la lectura de paramentos es difícil que arroje información debido al grado de deterioro que presentan, o a las numerosas restauraciones realizadas que han alterado y uniformizado la imagen de los lienzos de la muralla, la métrica subyacente es más difícil de alterar y posiblemente sea una de las escasas opciones que nos queden para poder profundizar en el conocimiento de la muralla de Segovia. (Ver esquema 2)

\section{Tipología de las torres de las murallas de Segovia.}

Como ya se ha comentado, atendiendo a la forma en planta de las torres de la muralla de Segovia, existen tres tipos de torres. La más extendida y abundante es la de planta rectangular que es la que predomina en prácticamente todo el trazado de la muralla. 
Si seguimos la numeración del Plan Director, podríamos considerar que esta tipología se inicia en la torre 18, adosada al actual teatro Cervantes, junto a la posición que ocupaba la puerta de San Martín.

Este primer sector, entre la casa de los Picos y la de las Cadenas, torres 18 a 24 , representa el frente del recinto amurallado hacia el resto de la ciudad de Segovia y tiene un ritmo de torres muy diferente al que se observa en el resto del trazado, quizá motivado por el alto escarpe natural que ya posee la roca, facilitando la defensa, y que se materializa claramente en el Azoguejo, donde el tramo elevado del acueducto en su entronque con la muralla, genera la estampa más famosa del monumento romano con un desnivel de casi 30 metros.

A partir de este punto, los desprendimientos en la base rocosa sobre la que se apoya la muralla, han hecho desaparecer un amplio sector de la muralla, observándose solo el arranque de algunas de las torres (la 25 y 25') ${ }^{5}$ estando el resto perdidas $(26,27,28,29,30)$, en unos lienzos muy rehechos y que transmiten poca autenticidad.

A partir del postigo de San Juan, encontramos nuevamente un tramo bastante largo, hasta la puerta de Santiago, torre 57, en el que ha desaparecido alguna de las torres, (la 34, 47, 48, 52', 53, 54 y 61) estando el resto muy retocadas y reconstruidas. Hasta la torre 44 , la topografía obliga a un zigzagueo en su trazado, por lo que su modulación presenta algunas irregularidades que habrá que estudiar más detenidamente.

El último sector, ya en la ladera sur, desde los jardines del Alcázar hasta el actual museo, desde la torre 73 hasta la 79. Este sector presenta una regularidad en sus proporciones, que se presenta como idóneo para los objetivos de este estudio, ignorando que las sucesivas restauraciones han alterado su imagen inicial, unificando los paños y dificultando una lectura constructiva de los paramentos de dichos lienzos, pero la métrica subyacente va resultar muy esclarecedora.

\footnotetext{
5 En ambos casos, posiblemente las torres originales apoyasen sobre la roca en la parte alta, por lo que los restos existentes actualmente posiblemente sean meros refuerzos de la roca realizados después del derrumbe, pero en ningún caso se trate de las bases de las torres originales.
} 
Menor difusión tienen las torres de planta circular, que se extienden desde la torre 9 a la 16, ambas inclusive, todas ellas en la cara sur, que constituye el sector correspondiente con el conocido como Salón de Isabel II, desde la puerta del Sol, el edificio de la Alhóndiga con sus dos torres y la torre siguiente, la número 16, situada en el jardín del actualmente conocido como Palacio del Conde Alpuente, esta última muy transformada con remates de fabrica de ladrillo, de aire historicista, posiblemente fruto de alguna reconstrucción.

Existe una excepción, la torre 59, completamente aislada en la ladera norte, reconstruida en el siglo $X X$ tras su desmorone. Se encuentra apoyada sobre un trozo de roca, completamente desgajada del resto por una falla geológica (Rodríguez Ortiz, 1998: 120). Esta circunstancia me induce a pensar que en el pasado pudo haber sufrido el mismo proceso de derrumbe, y se reconstrucción, perdiéndose su forma primigenia, pero en origen se trataba de una torre rectangular.

Fig. 3. Torre 59. Torre de planta circular, única excepción en la ladera norte. Se trata de una reconstrucción completa del siglo XX. Su arranque es de planta rectangular.

Respecto a las torres de planta poligonal, éstas se extienden desde la 80 hasta la $\mathrm{n}^{-}$8, disponiéndose de modo alterno en todo este tramo con otras de planta

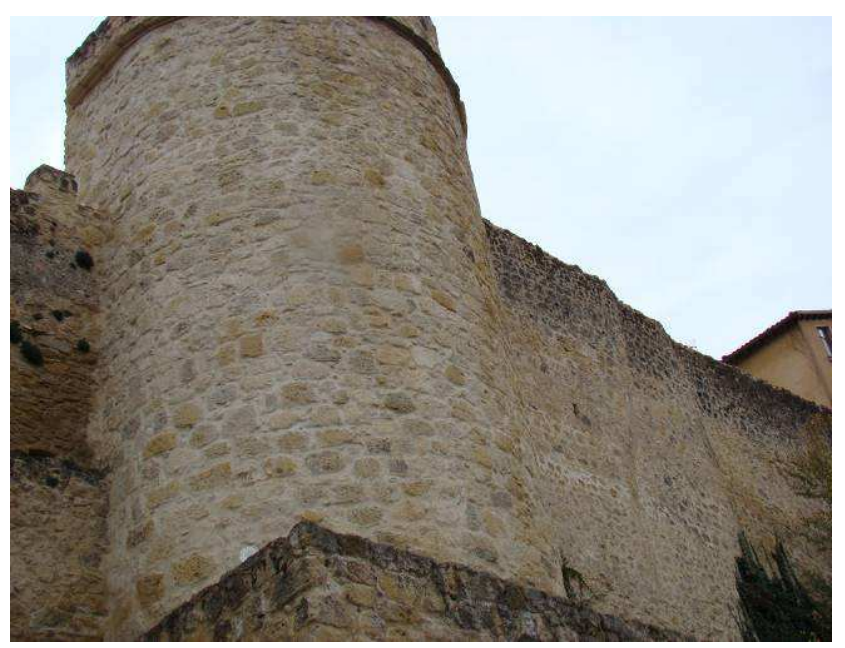
rectangular. Son poligonales, con diferente número de lados, la 81, 82, 83, 85, $1,4,6$ y $8^{6}$. Así frente a la aparente aleatoriedad y falta de regularidad que presentaba la disposición de las torres en lo relativo a su forma en planta, tras

${ }^{6}$ Como se ha indicado, se incluyen en este grupo las torres que en el Plan Director se consideran circulares. 
este nuevo enfoque, vemos que éstas se pueden organizar en tres sectores perfectamente agrupados, con las únicas excepciones que se han comentado de la torre 59 y las discontinuidades que suponen las torres desaparecidas, todas ellas en las caras norte y este. (Ver esquema 2)

- Rectangulares: De la 18 hasta la 79. Excepción: Torre 59 con las dudas expuestas.

- Circulares: de la 9 a la 16. La 59 excepción comentada.

- Alternancia de torres poligonales y rectangulares: De la 80 hasta la 8.

- Desaparecidas: 17, 25, 25', 26, 27, 28, 29, 30, 34, 52', 53, 54 y la 61.

Veamos cada uno de los sectores ${ }^{7}$.

\subsection{Sector 1 .}

Desde la Torre 72 a la 79. Largo aprox. 200 metros. Torres de planta rectangular, construidas en mampostería, con esquinas de sillería caliza. En algunos casos (74, 75, 78 y 79) presentan zócalo resaltado, con mismas características constructivas. Muy restauradas, por lo que su aspecto exterior es de mampostería. Las dimensiones de las torres son de 5, 20-5, 30 metros en el lado frontal por 2,65 metros de resalto, respecto al lienzo de la muralla.

Como puede observarse existe una gran regularidad geométrica entre las torres de este primer sector. La modulación que se obtiene dividiendo la longitud total del sector entre el número de lienzos existentes es de 25 metros, lo que supone una proporción lienzo/ancho de torre de 4,8 a 1 .

Inicialmente incluíamos en este sector a la torre 80, pero su métrica y sistema constructivo nos convencieron de que pertenece al sector 2. Del mismo modo, la torre 72, alterada por unos estribos laterales adosados que la desvir-

\footnotetext{
${ }^{7}$ En el estudio de la métrica de los lienzos y torres, no se ha tenido en consideración la altura de la muralla, dado que en muchos casos las partes altas están reconstruidas, por lo que la fiabilidad de dicha dimensión es prácticamente nula. Tampoco se ha tenido en consideración el espesor de los muros dado que esta medida solo puede tomarse allá donde existen adarves transitables, por lo tanto no ha sido posible efectuar una comparativa entre todos los sectores.
} 
túan así como la métrica del lienzo L72-73, nos inducen a incluirla en el sector correspondiente al Alcázar, que se ha excluido de este estudio.

\begin{tabular}{|l|rrr|}
\hline TORRE & TIPO & LARGO & ANCHO \\
\hline 73 & RECTANGULAR & 5,22 & 2,60 \\
74 & RECTANGULAR & 5,20 & 2,65 \\
75 & RECTANGULAR & 5,22 & 2,65 \\
76 & RECTANGULAR & 5,38 & 2,55 \\
77 & RECTANGULAR & 5,22 & 2,65 \\
78 & RECTANGULAR & 5,36 & 2,72 \\
79 & RECTANGULAR & 5,29 & 2,66 \\
\hline
\end{tabular}

\begin{tabular}{|l|r|}
\hline LIENZO & LARGO \\
\hline $73-74$ & 24,00 \\
$74-75$ & 19,30 \\
$75-76$ & 23,20 \\
$76-77$ & 16,82 \\
$77-78$ & 23,90 \\
$78-79$ & 21,57 \\
\hline
\end{tabular}

\subsection{Sector 2 .}

Desde la Torre 80 a la 9. Largo aprox. 420 metros.

Presenta una alternancia de torres de planta poligonal y torres de planta rectangular, entre las que hay que destacar varias singularidades que aparentemente desorganizan la regularidad de las medidas, si exclusivamente se miran los números presentados en las tablas adjuntas.

Las torres 81 y 82 flanquean el frontal del actual museo de Segovia, en el conocido como espolón. Esta disposición tan singular tiene claras connotaciones defensivas que justifican la alteración de la modulación para adecuarse a la orografía y las dimensiones del enclave a defender.

Por otra parte, la torre 2 se encuentra englobada en el conjunto de la puerta de San Andrés, podría considerarse toda la puerta como un único conjunto, flanqueada por la torre 1, prácticamente exenta, producto de una reforma posiblemente de la época de los Reyes Católicos. 


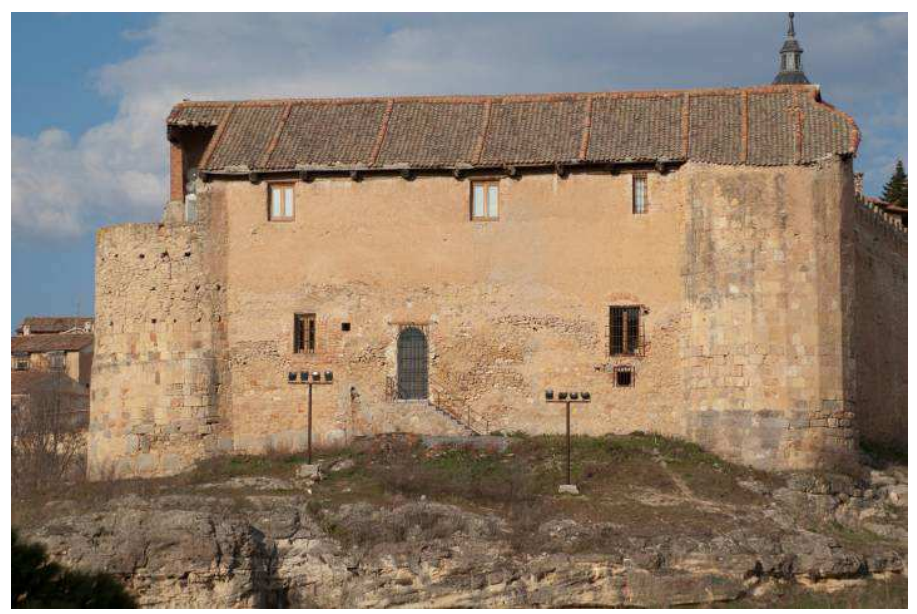

Fig. 4. Torres 81 y 82, flanqueando el edificio del actual museo de Segovia.

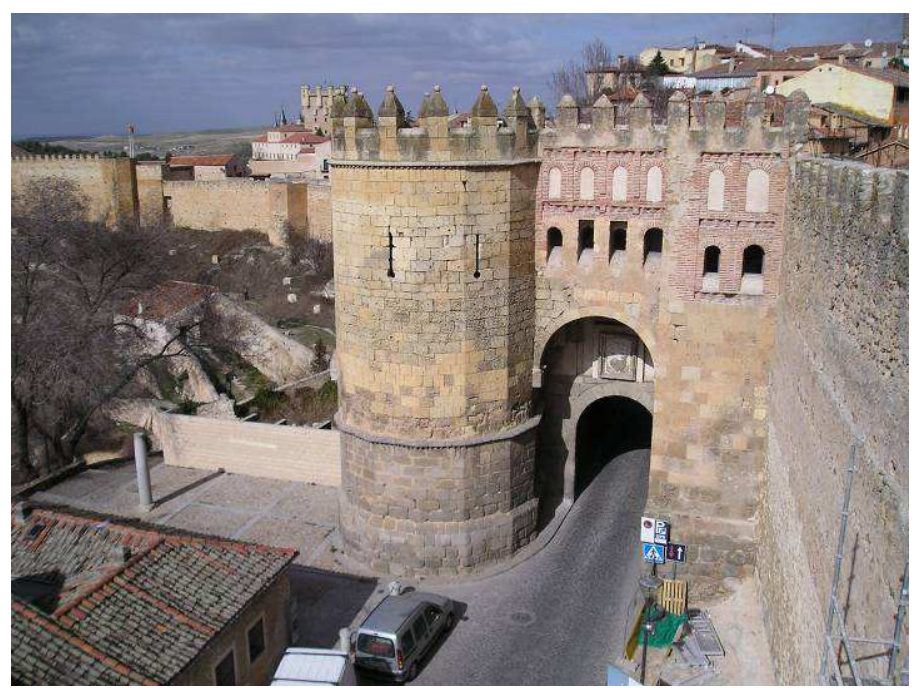

Fig. 5. Torres 1 (poligonal a la izquierda) y 2 (rectangular a la derecha), flanqueando la puerta de San Andrés. En vez de dos torres, puede concebirse el conjunto como una puerta, flanqueada por una torre poligonal.

Si tenemos en consideración estas aclaraciones, observaremos que se produce una alternancia, claramente intencionada, de torres poligonales y rectangulares. Además obviando estos lienzos mencionados, L81-82 y el de la puerta L1-2, las dimensiones de todos los demás son muy similares, en torno a los 34-35 metros, excepto el lienzo L2-3, de modo que la torre 3, se sitúa más próxima a la puerta de san Andrés, medida de carácter defensivo que se repite en todas las puertas de la muralla (San Cebrián, Santiago, y las desaparecidas de San Martín y San Juan). 
Las dimensiones de las torres rectangulares oscilan en torno a los 6 metros (siendo la torre 84 , reconstruida en el siglo $\mathrm{XX}$, la más pequeña, con 5,40 metros) por 3,70 metros de resalto (la torre 2 y la 7 solo 3,40 metros.)

Respecto a las torres poligonales ${ }^{8}$, señalar la similitud existente entre las 3 torres en disposición singular por una parte $(81,82$ y 1) y por la otra, las de las otras cuatro torres restantes (torres $85,4,6$ y 8 ). Resulta significativa la regularidad que presentan las tres torres 4,6 y 8 , que estilísticamente también presentan una unidad muy evidente y que las diferencia de las demás. Sus medidas son idénticas tanto en lo que se refiere a radio, longitud del lado y arco o ángulo que forma con los lienzos de muralla. Queda como excepción a este agrupamiento la torre 83 , cuya técnica constructiva también la diferencia completamente de todas las demás, lo que nos hace suponer que se trate de una reconstrucción completa, y posiblemente la forma que presenta en la actualidad, no era la original.

La modulación que se obtiene dividiendo la longitud total del lienzo entre 12 (el número de lienzos existentes contando linealmente) es de 34 metros, lo que supone una proporción lienzo/ancho de torre de 5,6 a 1, para una torre rectangular de 6 metros de ancho o una proporción de 5 a 1 para una poligonal de diámetro 6,80 metros.

Constructivamente podemos distinguir dos subsectores, siendo la frontera la puerta de san Andrés. Las torres de la decena de los 80 están ejecutadas con un basamento de sillería de caliza colocada a soga, y recrecidos de mampostería, a excepción de la 84 que se ha recrecido con ladrillo en el siglo XX.

El segundo subsector, se caracteriza por la presencia de sillería en los zócalos, generalmente resaltados y con abundante presencia de piezas de granito reutilizadas, posiblemente de origen romano ${ }^{9}$, mientras que se constatan re-

\footnotetext{
8 Para la toma de medidas de estas torres se ha hecho una toma de datos de cada una de ellas y se han circunscrito los polígonos resultantes en una circunferencia. Los datos que se introducen en la tabla son el radio de esta circunferencia, la longitud media de los lados de la torre y por último el arco que forman los diámetros que unen el centro de la circunferencia con los puntos de intersección de la torre con los lienzos de la muralla que acometen a ella.

${ }^{9}$ Se constata la presencia de lapidas funerarias romanas procedentes del expolio de los cementerios y sillares reutilizados de edificios y otras construcciones.
} 
crecidos de fabrica a base de arquillos decorativos de ladrillo, hasta la torre 5, a partir de la cual, los recrecidos son exclusivamente de mampostería. Hay que destacar que los zócalos de las torres 4 y 5 presentan restos de zarpas, elemento de carácter poliorcético de origen musulmán.

\begin{tabular}{|l|l|rr|r|rrr|}
\hline TORRE & TIPO & RADIO & LADO & N $^{\circ}$ LADOS & ARCO & LARGO & ANCHO \\
\hline 80 & RECTANGULAR & & & & & 5,86 & 3,50 \\
81 & POLIGONAL & 3,87 & 1,04 & 13 & $154^{\circ}$ & & \\
82 & POLIGONAL & 3,87 & 1,25 & 11 & $155^{\circ}$ & & \\
83 & POLIGONAL & 3,35 & 2,70 & 5 & $161^{\circ}$ & & \\
84 & RECTANGULAR & & & & & 5,40 & 3,72 \\
85 & POLIGONAL & 3,40 & 1,31 & 9 & $122^{\circ}$ & & \\
86 & RECTANGULAR & & & & & 6,13 & 3,77 \\
1 & POLIGONAL & 3,87 & 1,67 & 11 & $252^{\circ}$ & & \\
2 & RECTANGULAR & & & & & & 3,39 \\
3 & RECTANGULAR & & & & & 5,90 & 3,70 \\
4 & POLIGONAL & 3,40 & 1,30 & 9 & $169^{\circ}$ & & \\
5 & RECTANGULAR & & & & & 6,11 & 3,68 \\
6 & POLIGONAL & 3,40 & 1,33 & 8 & $172^{\circ}$ & & \\
7 & RECTANGULAR & & & & & 5,97 & 3,35 \\
8 & POLIGONAL & 3,40 & 1,32 & 8 & $170^{\circ}$ & & \\
\hline
\end{tabular}

\begin{tabular}{|lr|}
\hline LIENZO & LARGO \\
\hline $79-80$ & 33,28 \\
$81-82$ & 18,13 \\
$82-83$ & 33,80 \\
$83-84$ & 35,00 \\
$84-85$ & 34,00 \\
$85-86$ & 33,35 \\
$86-1$ & 33,43 \\
$1-2$ & PUERTA \\
$2-3$ & 25,95 \\
$3-4$ & 33,95 \\
$4-5$ & 35,00 \\
$5-6$ & 34,86 \\
$6-7$ & 35,01 \\
$7-8$ & 34,27 \\
$8-9$ & 34,92 \\
\hline
\end{tabular}




\subsection{Sector 3 .}

En el caso de las torres circulares, solo se analizan los lienzos y torres hasta el postigo de la Luna (ver nota 5). El conjunto queda reducido a pocos valores, pero se constata una elevada similitud de medidas. Por otra parte los valores son muy similares a los obtenidos para las torres poligonales, con un valor predominante para el radio de 3,40 metros. En este caso la separación entre torres es menor de lo que nos hemos encontrado hasta ahora, con la singularidad de que el lienzo entre las torres 10 y 11 es significativamente mayor que el resto de los existentes en este sector. La modulación media que obtenemos para estos lienzos es de unos 26 metros. En este caso la proporción entre torres y lienzos (26 metros de largo de lienzo a 6,80 metros de las torres) resulta un valor de alrededor de 3,8 a 1, lo que significa que la torres están relativamente más grandes y mas juntas.

\begin{tabular}{|l|lr|}
\hline TORRE & TIPO & RADIO \\
\hline 9 & CIRCULAR & 3,40 \\
10 & CIRCULAR & 3,00 \\
11 & CIRCULAR & 3,23 \\
12 & CIRCULAR & 3,57 \\
13 & CIRCULAR & 3,40 \\
\hline
\end{tabular}

Constructivamente todas se ejecutan en mampostería en la parte visible, ya que la mayor parte de ellas están coronadas por los edificios que se han construido encima, y han revocado sus paramentos. Sin embargo, en el lienzo L10-11, se observa un aparejo de sillares puestos en vertical (aparejo califal) de muy escasa altura, lo que nos induce a pensar que una parte del lienzo original puede estar enterrado bajo la cota actual del paseo del Salón. Se adjunta un cuadro resumen en el que se han incluido otros tramos de la muralla norte, aunque en esta zona los valores no son tan regulares, posiblemente debido a la topografía. Somos conscientes de que este cuadro tan escueto supone una simplificación excesiva, pero nos permite obtener algunas conclusiones, al obviar la profundización en la exactitud numérica ${ }^{10}$.

\footnotetext{
${ }^{10}$ En cualquier caso tampoco tiene mucho sentido entrar en dicha exactitud numérica dado que la muralla se construyó con una unidad de medida distinta del metro, que empleamos actualmente, posiblemente en pies y varas. Eso queda para un futuro estudio, que requerirá más exactitud en las medidas y otra finalidad en el planteamiento.
} 


\begin{tabular}{|c|c|c|c|c|c|c|c|}
\hline \multirow[b]{2}{*}{ SECTOR } & \multirow[b]{2}{*}{ TRAMO } & \multicolumn{3}{|c|}{ TORRES } & \multirow{2}{*}{\begin{tabular}{|l|} 
LIENZOS \\
MODULACION
\end{tabular}} & \multirow[b]{2}{*}{ PROPORCION } & \multirow{2}{*}{$\begin{array}{c}\text { SISTEMA } \\
\text { CONSTRUCTIVO }\end{array}$} \\
\hline & & FORMA & LARGO/RADIO & ANCHO & & & \\
\hline SECTOR 1 & $72-79$ & RECTANGULAR & 5,25 & 2,65 & 25 & $4,8-1$ & MAMPOSTERIA \\
\hline \multirow[t]{2}{*}{ SECTOR 2} & $80-1$ & ALTERNANCIA & Ǿ 3,87 - 6,00 & 3,70 & 35 & $5,8-1$ & SILLERIA/MAMP \\
\hline & $2-9$ & ALTERNANCIA & Ǿ 3,40 - 6,00 & 3,70 & 35 & $5,0-1$ & SILLERIA/LADRILLO \\
\hline SECTOR 3 & $10-13$ & CIRCULAR & Ǿ 3,40 & & 26 & $3,8-1$ & MAMPOSTERIA \\
\hline \multirow[t]{3}{*}{ SECTOR 4} & $31-42$ & RECTANGULAR & 5,30 & 2,20 & 38 & $7,0-1$ & MAMPOSTERIA \\
\hline & $43-47$ & RECTANGULAR & 5,30 & 2,05 & 24 & $4,5-1$ & MAMPOSTERIA \\
\hline & $47-52$ & RECTANGULAR & 5,20 & 2,15 & 27 & $5,4-1$ & MAMPOSTERIA \\
\hline
\end{tabular}

Las diferencias entre los distintos tramos de torres rectangulares no son tantas, a pesar de la distancia existente entre unos y otros, de una punta a otra de la ciudad. Los tamaños de las torres, son en todos los casos muy próximos a los 5,20-5,30 metros, el resalto es menor en la cara norte, posiblemente motivado por la cercanía del talud vertical, que desde el punto de vista defensivo lo hace más innecesario y deja menos sitio para ello. Constructivamente, son muy similares, (afirmación que debe entenderse como una generalización, ya que existen numerosas matizaciones cuyo estudio excedería el alcance de esta comunicación). La modulación de los lienzos y la proporción lienzo/torre, también son parecidas, en torno a los 25 metros y al 5-1, respectivamente. Tan solo el tramo 31-42 presenta una separación mucho mayor entre sus torres, que lo diferencia de los demás ${ }^{11}$.

Pero nos centraremos en los sectores 2 y 3 . Las torres son de una forma en planta distinta, pero también sensiblemente más grandes que las rectangulares. Constructivamente son muy diferentes, más elegantes, especialmente las del tramo 2-9, y más pobres, aparentemente, las de planta circular. La diferenciación se refuerza con más datos. La modulación de los lienzos del sector 2 es considerablemente mayor, mientras que las torres del sector 3, a pesar de ofrecer una modulación similar a las rectangulares, evidencian una proporción (de torres más grandes y mas juntas) que las contrasta con el resto. Es significativo que en esta tipología las torres se disponen mucho mas juntas, excepto el lienzo central (el 10-11) en el que las torres se separan hasta valores similares al 5 a 1 .

${ }^{11}$ Todo este conjunto de valores y proporciones, se justifican en base a la tecnología militar y a la poliorcética del momento histórico en que se construyen cada muralla. 


\section{Conclusiones.}

En la muralla de Segovia, existen tres tipologías de torres claramente diferenciadas desde el punto de vista morfológico, unas de planta rectangular, las más numerosas, otras de planta poligonal, que se concentran en un sector de la ladera sur, premeditadamente intercaladas con torres de planta rectangular y por último, el tercer grupo de planta circular, existente tan solo en un sector del alzado sur de la muralla. Hemos encontrado matices, como el hecho de que en las proximidades de las puertas, los lienzos sean más cortos, de modo que las torres se sitúan más cerca de la puerta, para contribuir a su mejor defensa. Aun más importante, frente a la aleatoriedad que aparentemente existía en los diseños y disposición de las torres de la muralla, se constata, que se agrupan en tres sectores. (La zona norte, es más irregular en las modulaciones como se ha indicado, posiblemente se deba exclusivamente a la topografía, porque en forma, métrica y sistemas constructivos, se asemeja considerablemente al sector 1 ).

De esta forma, se entiende que existen, al menos, tres fases constructivas de los lienzos de la muralla segoviana, que se corresponden con tres sectores homogéneos, nítidos y perfectamente diferenciados en el alzado sur. Con este estudio, creemos haber demostrado que no se trata de una muralla realizada de una sola vez de manera unitaria, sino que se trata de al menos tres "murallas" con morfologías, sistemas constructivos, dimensiones, modulaciones y proporciones diferentes. Aunque, como se ha indicado, no hay datos arqueológicos asociados, todo parece indicar que se trata de tres cronologías diferentes, o tres murallas diferentes subyacentes, aunque las intervenciones realizadas a lo largo de tiempo haya uniformizado su aspecto en la actualidad.

¿Nos encontramos ante restos reutilizados de otras murallas anteriores? ¿Puede ser que la muralla medieval esté aprovechando unos cimentos preexistentes, ahora ocultos? ¿Por qué una morfología, una métrica y un sistema constructivo diferentes? ¿Puede tratarse de parte de una muralla de la Segovia musulmana? La presencia de otros detalles de influjo andalusí (zarpas, aparejo califal, etc.) podrían inducirnos a pensarlo. No tenemos datos asociados para asignarlo una cronología fiable.

¿Y el sector de torres circulares? ¿Puede haber subyacente una modulación romana bajo la muralla que vemos actualmente? 
Como se ha indicado, hasta la fecha no hay suficiente información arqueológica que permita asignar una cronología a cada una de las tipologías. Si bien, una muralla no es la construcción más apropiada para la búsqueda de una métrica constructiva exacta, posiblemente si la estudiamos con mayor detenimiento encontremos que existe una unidad de medida distinta para cada una de los tres tipos sectores tipológicos en que hemos diferenciado la muralla (pies castellanos, pies capitolinos, codos musulmanes, etc.).

¿Existe un único módulo numérico constructivo o existen varios? ¿Existe alguna relación entre la tipología de torre y el módulo constructivo numérico existente en su fábrica? El empleo de las medidas antropológicas, puede desvelarnos más datos sobre estas incógnitas. Es necesario realizar un estudio en busca de las unidades de medida con que están realizadas cada una de las tipologías de torre. Como se ha indicado, puede que sea una de las escasas opciones que nos queden para poder profundizar en el conocimiento de la muralla de Segovia. Seguiremos investigando.

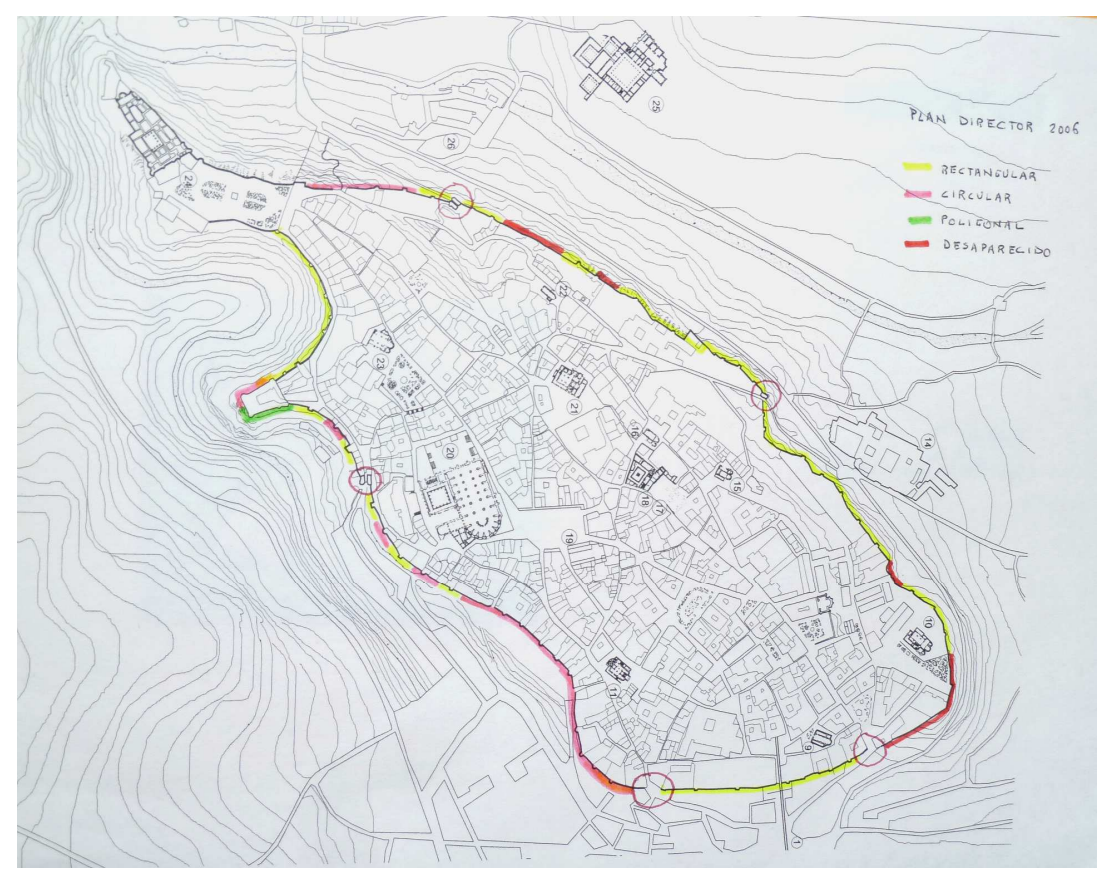

Fig.6. Esquema 1 - Plano de tipología de torres de la muralla, según el Plan Director de 2006. 


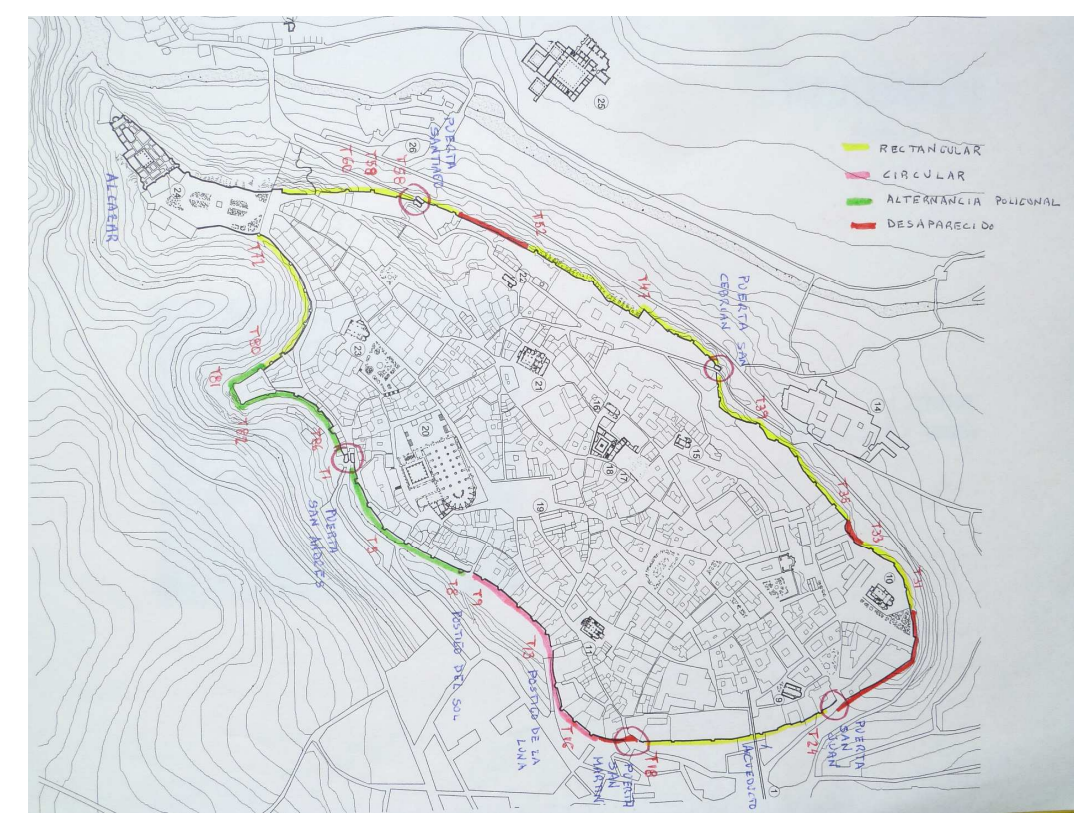

Fig. 7. Esquema 2 - Plano de tipología de torres de la muralla, según nuestro estudio.

\section{Bibliografía.}

AVRIAL y FlORES, J. M. (1953): “Segovia pintoresca.” Estudios Segovianos. Tomo V, no13 y 14, págs. 29-73. Real Academia de San Quirce, Segovia.

Chaves Martín, M. A. (1998): Arquitectura y urbanismo en la ciudad de Segovia (1750-1950). Cámara de la Propiedad Urbana, Segovia.

_ (2003): Puertas de la muralla de Segovia. Asociación Cultural Plaza Mayor de Segovia, Segovia.

RuIZ HeRnANDO, J. A. (1982): Historia del urbanismo en la ciudad de Segovia del siglo XII al XIX. Diputación provincial de Segovia, Segovia.

_ (1986): La ciudad de Segovia. Ayuntamiento de Segovia, Segovia.

VV.AA. (1991): Segovia 1088-1988. Actas del Congreso de Historia de la Ciudad. Academia de Historia de San Quirce, Segovia.

VV.AA. (2010): Curso de Historia de Segovia. Ayuntamiento de Segovia, Segovia. 


\section{Fuentes Documentales}

Ayuntamiento de Segovia. Concejalía de Patrimonio Histórico.

FERNÁNDEZ-LONGORIA AlCÁNTARA, P. (2006): Plan Director de las murallas de Segovia.

Martín Blanco, M. A. 2009 a. Proyecto de restauración de la muralla de Segovia Fase I. Ronda de Juan II.

Martín Blanco, M. A. 2009 b. Proyecto de restauración de la muralla de Segovia Fase II. Muralla norte.

Martín Blanco, M. A. 2010 a. Proyecto de Paso de inspección en el paño norte de la muralla de Segovia.

MARTín Blanco, M. A. 2010 b. Proyecto recuperación de la muralla y su entorno urbano. Plaza del Socorro.

Archivo Municipal de Segovia.

RodríGuez OrTIZ, J. M. (1998). (Dir) Estudio Geotécnico. Sobre los problemas de inestabilidad que afectan al recinto amurallado de la ciudad de Segovia. 